\section{SSRL enters its fourth decade with SPEAR-3}

We reported earlier this year [J. Synchrotron Rad. (2004), 11, 214] details of the SPEAR upgrade to SPEAR-3, which has turned one of the pioneering storage-ring facilities into a third-generation synchrotron radiation source. The $3 \mathrm{GeV} 500 \mathrm{~mA}$ ring, whose lattice configuration was constrained to maintain the existing beamline alignment and $234 \mathrm{~m}$ circumference racetrack geometry of SPEAR, has a low emittance of $12 \mathrm{~nm}$ rad, providing a third-generation light source capability for the SSRL users. Despite the emergence of two other third-generation sources, one in the Bay area and the other in Chicago, SSRL has remained a major contributor to synchrotronradiation-based research.

Two eight-pole electromagnet wigglers have been replaced by 20 pole permanent-magnet devices. SPEAR-3 has the capacity to accommodate a number of future beamlines - up to 14 new bends and seven new IDs (not including possible chicane configurations). The first $100 \mathrm{~mA}$ fill was reached on 22 January 2004. The following weeks were devoted to refined lattice tuning optimization, beam property characterization and vacuum conditioning, while awaiting radiation safety approval to open the X-ray beamlines. Beamline 9, the first to be given safety approval for SPEAR-3 operation, was opened on 8 March.

The first users began collecting data on SPEAR-3 on 15 March 2004. Robert Hettel, head of the accelerator department said that, as of early June 2004, beam has been delivered to users with an average of greater than $95 \%$ of the scheduled time since the beginning of the user run. Beam lifetime has been steadily improving, with a $30 \mathrm{~h}$ lifetime at $100 \mathrm{~mA}$ in early June. Currently, SPEAR-3 is filled three times a day, with currents starting at $100 \mathrm{~mA}$ falling to around $80 \mathrm{~mA}$ over a typical $8 \mathrm{~h}$ period. Beam injection is very quick, normally a few minutes, due to at-energy injection. Users are reporting high-quality data, and a slow orbit feedback system responding to readings from beam position monitors mounted on temperature-stable Invar supports maintain superb micrometre-level stability. Keith Hodgson, Director of SSRL, said that SPEAR-3 will continue to operate at $100 \mathrm{~mA}$ during its first commissioning run (March-July 2004); the installation of additional shielding in summer 2004 will enable $500 \mathrm{~mA}$ operation in future run cycles.

Having steered the upgrade (essentially a new machine), Keith Hodgson is now giving a high priority to implementing a 'top-off' injection mode with beamline photon stoppers open. Improving beam stability for users will be an on-going goal for machine development, with near-term plans for increasing the number of beam position monitors and the speed of the orbit feedback system towards $200 \mathrm{~Hz}$ already underway. SPEAR-3 has brought new light and new

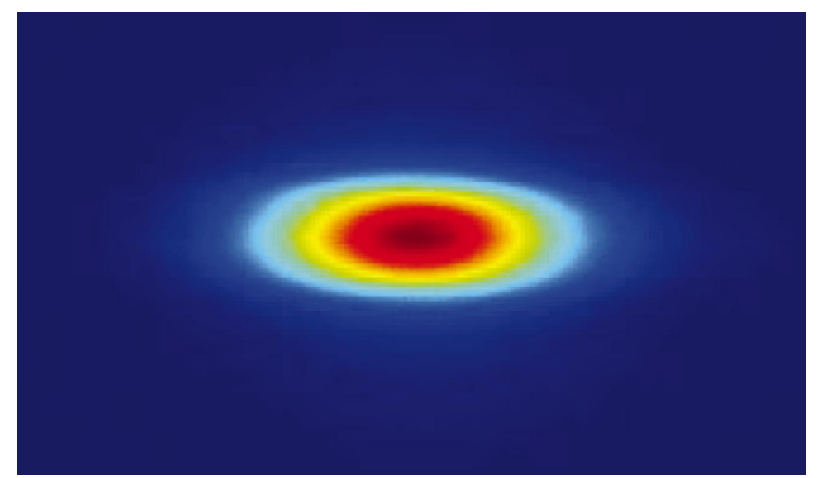

Pinhole image of the SPEAR-3 beam from a dipole source point, having dimensions of $\sim 50 \times 150 \mu \mathrm{m}$ r.m.s. life to the already fruitful scientific program at SSRL, serving its already established and growing community of synchrotron light users. SPEAR-3 enables new experimental capabilities and opportunities for new science that were unobtainable with the SPEAR-2 second-generation light source. SPEAR-3 will continue to be the workhorse research engine for SSRL, operating in parallel with the LCLS X-ray free-electron laser which is expected to come on-line late in 2008 at SLAC.

\section{Daresbury gets a new director}

Professor Colin Whitehouse took over the Directorship of the Daresbury Laboratory on 1 May 2004, after joining CCLRC as Director of Engineering in May 2003. Prior to joining CCLRC, he was Pro-Vice-Chancellor for Research and Research Exploitation at the University of Sheffield since 1999 . He had joined the University as Professor of Electronic Materials in 1993 where he became Director of the University's EPSRC National Centre for III-V Semiconductor Technologies and Head of its Electronic and Electrical Engineering Department in 1994. His interest in semiconductor research has its origins from his $\mathrm{PhD}$ which he undertook at GEC Hirst Research Centre during 1974-1977. Colin has authored over 290 journal papers and conference presentations relating to his research. For many years he has been involved with synchrotron radiation research and has used SRS and ESRF extensively.

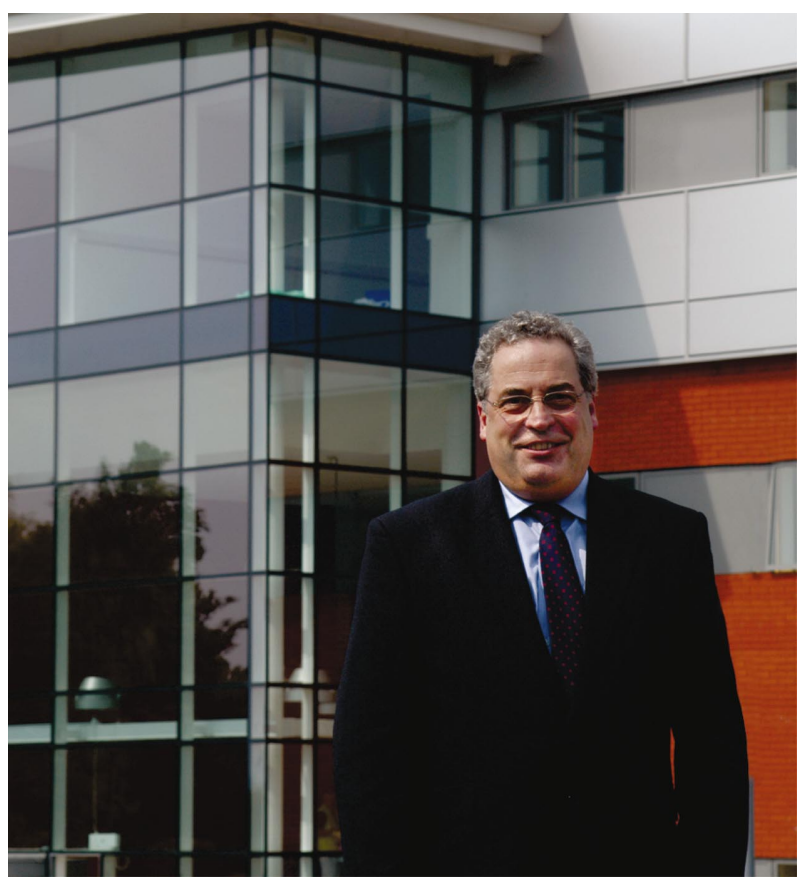

Colin Whitehouse in front of one of the new buildings on the Daresbury campus.

Colin also chaired the 'White Rose' Research Committee during 1994-1997, which facilitated numerous new and highly successful research collaborations between Sheffield, Leeds and York Universities, and also contributed significantly to the Regional Development Agency (Yorkshire Forward) strategic planning processes to stimulate regional economic development. This experience appeared to have helped him in rapidly becoming very active in the north-west region driving forward major new ambitious plans for the Daresbury 